\title{
Stress Echocardiography: Diagnostic, Prognostic, and Management Tool for the 1990s
}

\author{
WILLIAM F. ARMSTRONG, M.D. \\ Department of Cardiology, University of Michigan Medical Center, Ann Arbor, Michigan
}

The coming decades will provide dramatic new challenges for those of us in clinical cardiology. We will be challenged to provide more accurate and more clinically pertinent information in a more cost-effective manner for patients ranging in age from their teens to their nineties. The spectrum of diseases will range from congenital to valvular to ischemic, with the latter predominating. As such, our management tools must evolve over the coming years if we are to remain competitive as a cost-effective means of evaluating and managing patients with known or suspected cardiac disease. The following articles all deal with stress echocardiography, a technique that I feel will clearly play a predominant role in noninvasive cardiology in fulfilling these crucial goals for the next decade.

The following articles have been solicited and arranged to provide an extensive background in the techniques of stress echocardiography, the hypotheses underlying their utilization in different patient subsets, a state-of-the-art review of the methods of analysis, and an up-todate critique of their diagnostic utility.

Among the modern imaging techniques dealing with coronary artery disease, two-dimensional echocardiography clearly is one of the simplest. Its background dates to the observation of Tennant and Wiggers that wall motion becomes abnormal almost immediately after the onset of myocardial ischemia. Two-dimensional echocardiography merely provides a modern day method for detecting and quantify- ing that abnormality. The magnitude of abnormal wall motion is directly proportional to the amount of ischemic myocardium, and as such provides valuable information regarding patient prognosis. The ease with which the abnormality becomes manifest also provides prognostic information regarding the likelihood of future events.

Stress echocardiography has several advantages for the cost-conscious 1990s. It is one of the most widely disseminated cardiac imaging technologies available. More importantly, it is clearly the most versatile imaging technique we have for cardiac disease. As two-dimensional echocardiography and Doppler are capable of diagnosing virtually any form of anatomical heart disease, any entity that masquerades as or coexists with coronary artery disease is accurately detected. This alone confers a tremendous advantage compared to the relatively limited versatility of other imaging techniques. The cost of two-dimensional echocardiography compared to other techniques is obviously lower and it has no known risks. Stress echocardiography can be used with a substantial increase in convenience to the patient, as results are available almost immediately and the total time commitment on the part of our patients exceeds that of the routine treadmill examination by no more than 30 minutes. All of these factors combined clearly make stress echocardiography one of the predominant provocative testing tools for the 1990s and one with which we should all definitely become intimately familiar. 